\title{
A Case of Spontaneous Tumor Lysis Syndrome in Malignant Melanoma
}

\author{
Nobumichi Takeuchi ${ }^{\mathrm{a}, \mathrm{d}}$, Shun Miyazawa ${ }^{\mathrm{a}}$, Zentaro Ohno ${ }^{\mathrm{a}}$, Sonomi Yoshida ${ }^{\mathrm{a}}$, Tetsu Tsukamoto ${ }^{\mathrm{b}}$, \\ Masayuki Fujiwara ${ }^{\mathrm{c}}$
}

\begin{abstract}
A 62-year-old man with a complaint of back pain lasting 2 months was admitted. He also presented a huge abdominal tumor. Diagnostic imaging showed metastatic tumors in the liver, lumbar vertebrae and bilateral lung. An ultrasound-guided needle biopsy revealed a lung tumor containing melanic tissue. Subsequently, there was an evident elevation in uric acid, phosphoric acid, potassium and lactate dehydrogenase concentrations in serum. Continuous hemodiafiltration and administration of rasburicase was initiated following the diagnosis of tumor lysis syndrome (TLS). However, he died on the fourth day owing to arrhythmia. An autopsy revealed metastatic deposits in the liver, lung, spine, ribs, and lymph nodes along the biliary system. Microscopic examinations revealed massive necrosis of normal hepatocytes and tumor cells with disseminated tumor thrombi in the portal system. The catastrophic progression of TLS appears to be influenced by a persistent portal blood flow deficiency by portal tumor thrombus in this case.
\end{abstract}

Keywords: Spontaneous tumor lysis syndrome; Malignant melanoma; Metastasis; Arrhythmia

\section{Introduction}

Tumor lysis syndrome (TLS) is a critical condition involving oncological emergency and usually appears after the administration of treatments associated with massive tumor cell death [1]. Spontaneous TLS occurs in the absence of chemotherapy.

Manuscript accepted for publication May 09, 2016

aDepartment of Medical Oncology, Ina Central Hospital, 1313-1 KoshirouKubo, Ina-city, Nagano 396-8555, Japan

bepartment of Pathology, Tokyo Medical University Hachiouji Medical Center, 1163 Tatemachi, Hachiouji, Tokyo 193-0998, Japan

'Department of Pathology, Ina Central Hospital, 1313-1 Koshirou-Kubo, Inacity, Nagano 396-8555, Japan

${ }^{\mathrm{d} C}$ Corresponding Author: Nobumichi Takeuchi, Department of Medical Oncology, Ina Central Hospital, 1313-1 Koshirou-Kubo, Ina-city, Nagano 396-8555, Japan.Email: noblesse11@gmail.com

doi: http://dx.doi.org/10.14740/wjon970w
Despite the fact that spontaneous TLS among various malignancies has been sporadically reported, it has only been recognized in rare cases, and its etiology remains unclassified [2,3].

TLS onset after commencing treatment becomes a complication avoided by the risk stratification and prophylactic use of rasburicase or allopurinol prior to treatment $[4,5]$. However, spontaneous TLS remains very difficult to overcome.

Here we present a case of spontaneous TLS in a male with a later diagnosis of malignant melanoma revealed by autopsy.

\section{Case Report}

A 62-year-old male with a complaint of severe back pain persisting for 2 months was admitted to our hospital. Hindered by the aggravation of the pain, the patient could not lie on his back for several days. A chest X-ray revealed a right lung tumor that was confirmed as a well-defined mass of $2 \mathrm{~cm}$ in diameter by computed tomography.

He had no remarkable medical history except for a food allergy to buckwheat and type II diabetes mellitus for 2 years, which was stable and treated without medication.

In addition, he had no particular family history of malignant disease or habit of smoking.

He was alert and exhibited extreme hepatomegaly with mild tenderness and evident dehydration with moderate jaundice and edema on the lower extremities. An asymmetrical, irregular, bordered, black and slightly elevated lesion was found in the right lower abdominal wall, which stabilized at a size of $2 \mathrm{~cm}$ in diameter for several years.

The blood test showed liver dysfunction and pre-renal dehydration. The circulating white blood cells were 13,440 cells/ $\mathrm{mL}$, hemoglobin was $12.4 \mathrm{~g} / \mathrm{dL}$ and the platelet count was 27.8 $\times 10^{4} \mathrm{cells} / \mu \mathrm{L}$. The chemistry panel showed a potassium level of $5.1 \mathrm{mmol} / \mathrm{L}$, chloride of $99 \mathrm{mmol} / \mathrm{L}$, phosphate of $3.6 \mathrm{mg} /$ $\mathrm{dL}$ and elevated levels of lactate dehydrogenase (LDH), aspartate aminotransferase (AST), alanine aminotransferase (ALT), alkaline phosphatase (ALP), total bilirubin (TB), direct bilirubin (DB) and C-reactive protein (CRP). Computed tomography and MRI revealed disseminated metastatic changes in lumbar spinal (Fig. 1), well-defined pulmonary tumors in the right lower and left upper lobes (Fig. 2) and an ambiguously bordered low-density area of the liver, predominantly in the left lobe as well as a moderate volume of ascites (Fig. 3). 


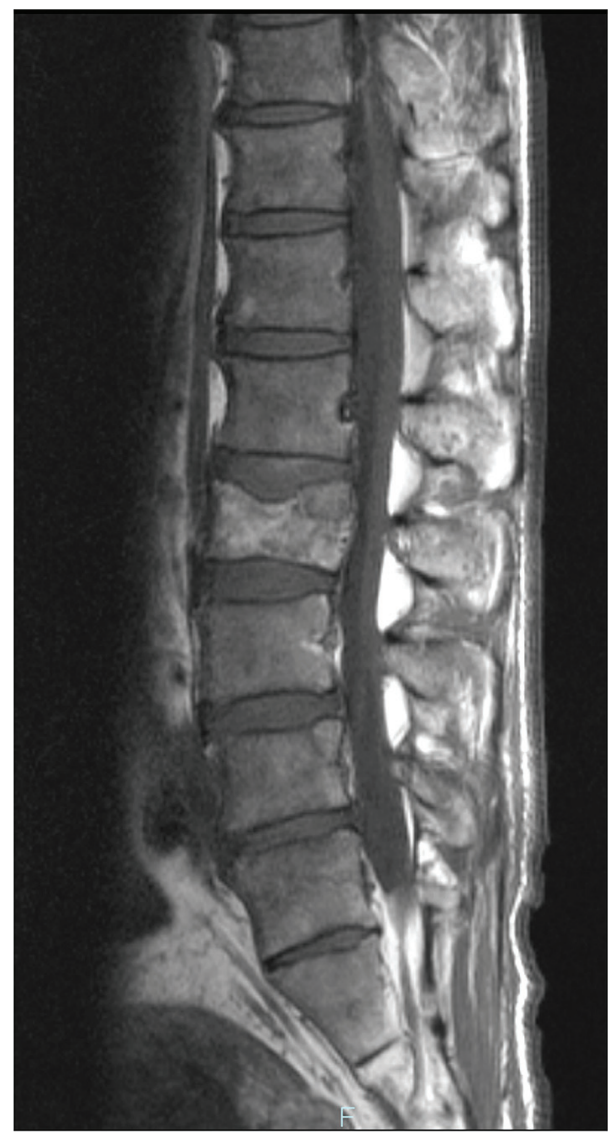

Figure 1. Spinal MRI. T1-weighed MR image showed metastatic lesions spread widely in the vertebral bodies.

Rapid infusion with oxycodone to resuscitate his dehydrated status and back pain improved his general status and back pain, except for oliguria on the second day of admission. On the third day, an ultrasound-guided needle biopsy of the right lung tumor revealed its melanic contents. Then he was transferred to an intensive care unit for continuous hemodia-

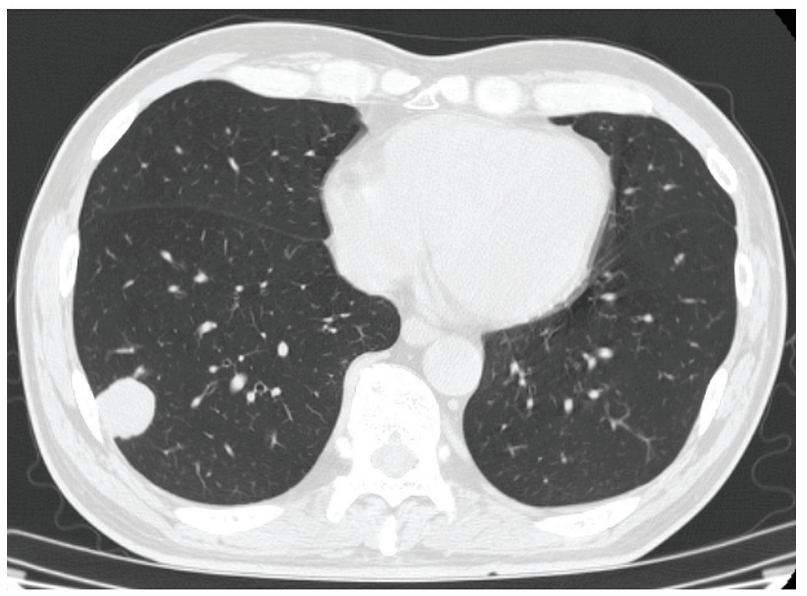

Figure 2. Chest CT. A well-defined right lung tumor of $25 \mathrm{~mm}$ in diameter.

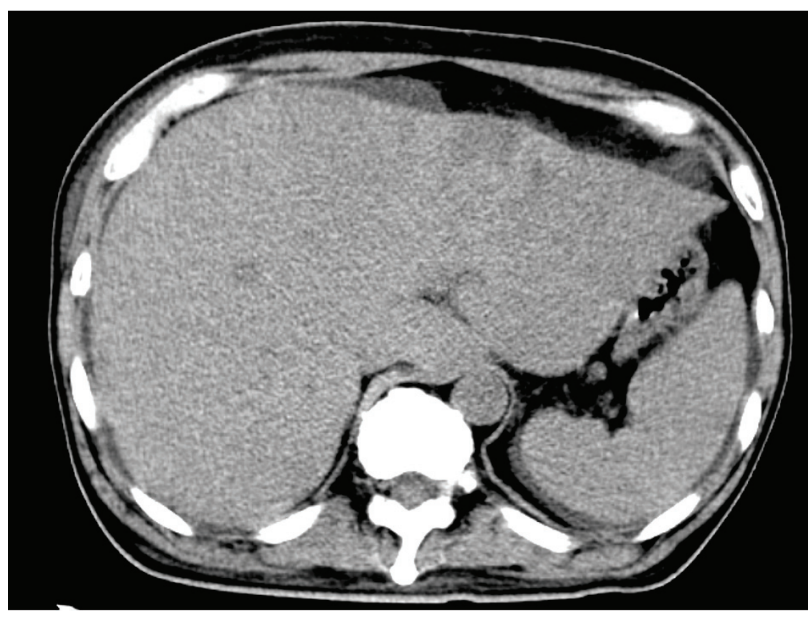

Figure 3. Abdominal CT. Diffuse hypo-density area in the swelled liver with ascites.

filtration for treating spontaneous TLS, which was diagnosed by a blood test that morning. Indexes of TLS during clinical course are shown in Table 1.

Rasburicase administration and other treatments for progressive metabolic acidosis were performed with rapid infusion.

Despite all the intensive treatments, the patient experienced ventricular fibrillation and a sudden cardiac arrest, and he expired $66 \mathrm{~h}$ after admission.

\section{Pathological findings}

The skin lesion in the right abdominal wall suspicious of primary malignant melanoma did not exhibit atypical melanocytes.

The needle biopsy of left lung tumor performed $36 \mathrm{~h}$ before the patient's death revealed a diffuse invasion of melanocytes without massive necrosis. Immunohistochemical analysis showed positively stained tumor cells by S100, HMB45 and MART1. The Ki-67 staining rate was 30\%. The molecular biological examination revealed a positive BRAF V600E mutation.

The autopsy reported a blackish swollen liver, 3,600 $\mathrm{g}$ in weight (Fig. 4), disseminated blackish tumors of the bilateral

Table 1. Indexes of Tumor Lysis Syndrome During Clinical Course

\begin{tabular}{llll}
\hline & \multicolumn{3}{c}{ Time after admission (h) } \\
\cline { 2 - 4 } & $\mathbf{0}$ & $\mathbf{3 6}$ & $\mathbf{5 8}$ \\
\hline T.Bil (mg/dL) & 4.34 & 6.71 & 9.63 \\
LDH (IU/L) & 8,756 & 20,223 & 52,700 \\
\hline UA (mg/dL) & 8.5 & 13.9 & 0.9 \\
P (mg/dL) & 3.6 & 6.8 & 12.6 \\
K (mEq/L) & 5.1 & 6.2 & 7.9 \\
Ca (mg/dL) & 9.4 & 8.2 & 7.9 \\
\hline
\end{tabular}




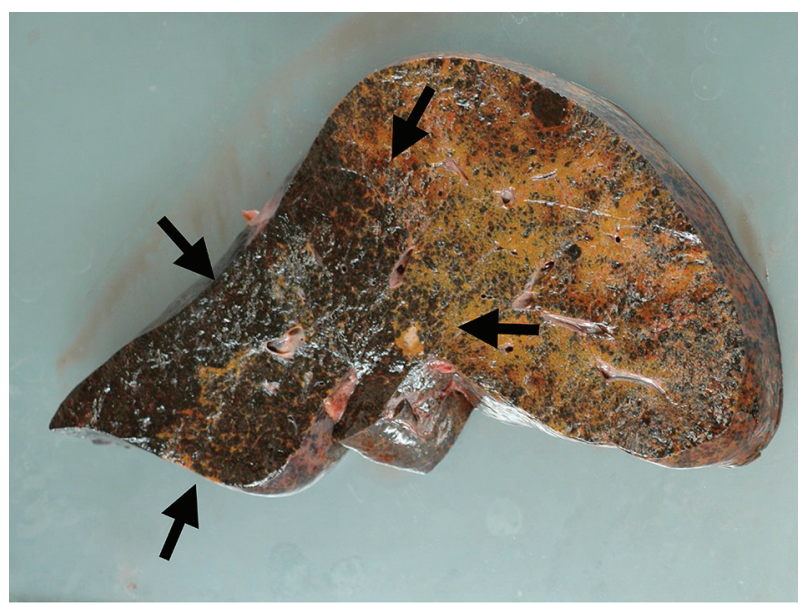

Figure 4. Macroscopic view of liver cut surface. Blackish swelled liver showed diffuse metastatic melanocyte invasion (arrows).

lungs (Fig. 5) and widely spread metastatic lesions in the vertebrae (Fig. 6), ribs and lymph nodes in the abdominal and thoracic cavity as well as the mediastinum, submucosa of the stomach and spleen. Massive tumor cell emboli were observed in the main trunk of the portal vein and its branches within a wide area, including the epigastric vessels.

Microscopic examination of these organs revealed several interesting findings. Massive necrosis of normal hepatocytes and melanoma cells was observed in the liver. The lesion was composed of two parts: 1) centrilobular necrosis without bleeding; and 2) perilobular necrosis with hemorrhage (Fig. 7). Extensive necrosis was observed within the largest pulmonary tumor (Fig. 8), unlike the biopsied specimen taken antemortem (Fig. 9).

\section{Discussion}

TLS represents an oncological emergency involving a collec-

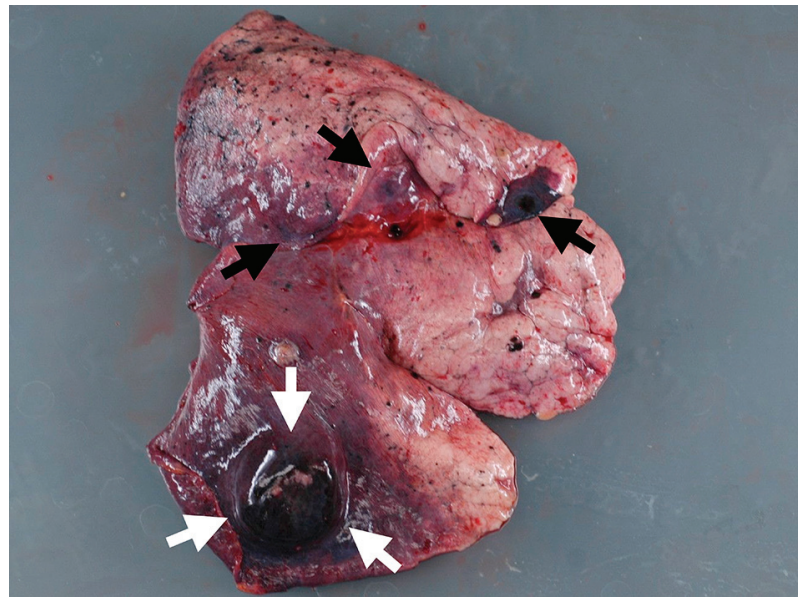

Figure 5. Macroscopic view of lung lesion. Well-defined metastasis (white arrows) was accompanied with multiple metastases (black arrows).

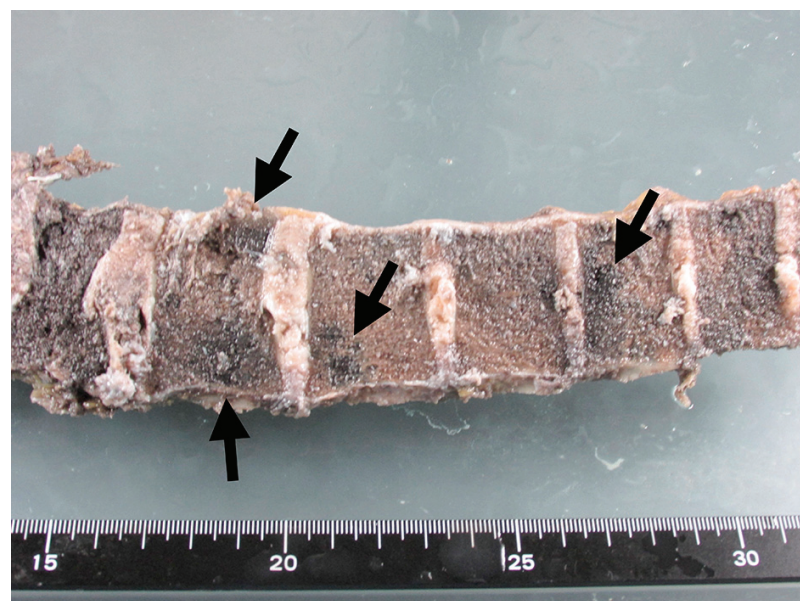

Figure 6. Macroscopic view of lumber spine. Metastatic lesions were spread in the vertebrae (arrows).

tion of metabolic and electrolyte disturbances that can be observed during the destruction of tumor cells [6]. It is commonly recognized as a severe complication of an excellent response to treatment for hematological malignancy, but the reports of TLS in solid tumors have accumulated only recently.

Spontaneous TLS remains a rare disease and is difficult to diagnose and treat as the etiology and pathogenesis are unknown. However, there are a small number of case reports on spontaneous TLS even in solid tumors.

To the best of our knowledge, there are eight existing case reports of TLS in malignant melanoma, including one spontaneous case of TLS without an autopsy [7-14].

Therefore, our report is the first autopsied case of spontaneous TLS caused by malignant melanoma.

Hands et al advocated that diagnostic criteria for laboratory and clinical TLS should be respectively considered based on the presence of clinical symptoms of seizure, arrhythmia, cardiac arrest and the elevation in creatinine con-

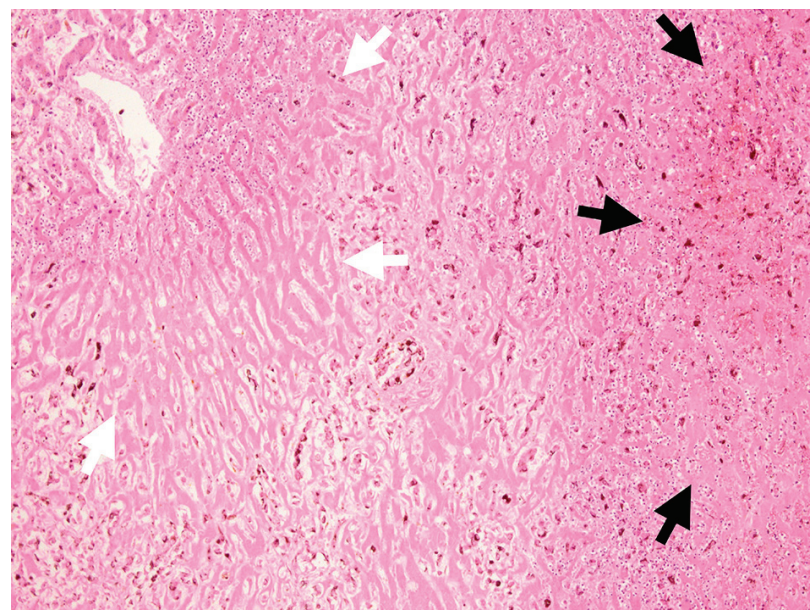

Figure 7. Microscopic view of liver cut surface. Centrilobular necrosis without bleeding (white arrow) and perilobular necrosis with hemorrhage were shown (black arrow). Diffuse invasion of atypical melanocytes was observed. 


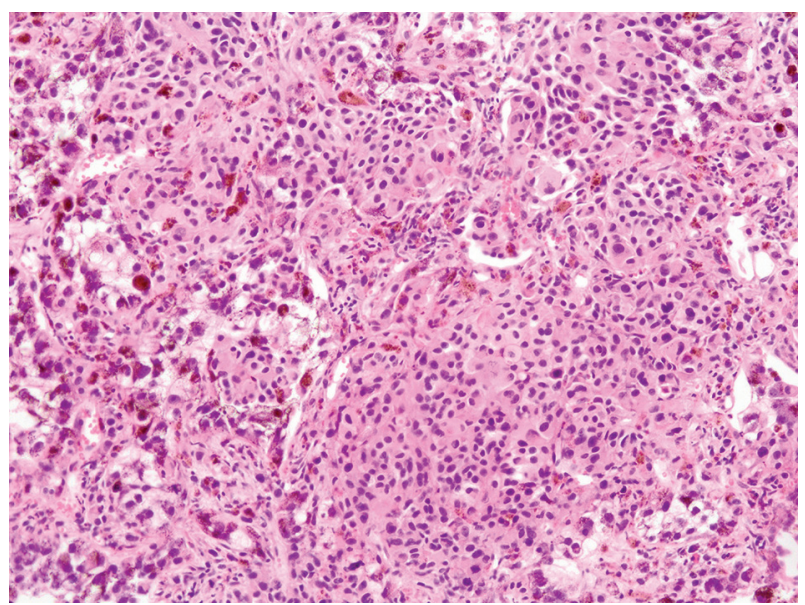

Figure 8. Microscopic view of lung lesion on autopsy. Lung metastasis with diffuse necrosis taken by autopsy was shown.

centration in serum in 1993 [15]. In 2004, sophisticated TLS diagnostic criteria were presented by Cairo and Bishop [4], and in 2010, an expert TLS panel consensus was published, which at present, is widely used for diagnostic purposes. The consensus categorizes the risk of TLS after the initiation of treatment using three levels judged by tumor volume and chemosensitivity [16]. Gemici added that risk factors should also be considered (e.g., the presence of renal dysfunction, liver metastasis, dehydration, infection and elevation in serum concentration of LDH or uric acid) [17]. For a hematological malignancy, the risk can be subdivided by the type of disease. However, the etiology of the solid tumor is uncertain owing to its rarity.

In our case, upon admission, the patient did exhibit a slight elevation in potassium and uric acid levels but no elevation in phosphate levels. Considering that the general dehydration was caused by poor oral intake of water during the previous weeks, TLS was difficult to assess. However, both the high elevation in LDH levels and large liver tumor were risk factors for TLS. Forty hours after his admission, LDH, AST and ALT levels increased by a factor of $\geq 2$, and uric acid and phosphate levels were also elevated by approximately double the levels at admission.

We suspect that the patient had subacute hepatic cell necrosis, which was caused by tumor emboli of the portal vein and hepatic artery without tumor lysis at the time of admission. The disease catastrophically progressed into acute spontaneous tumor lysis within $40 \mathrm{~h}$ of admission.

The results of the microscopic examination of the lungbiopsied specimen performed antemortem and the autopsied organ clearly present the details. Pulmonary tumor necrosis, which is shown in the autopsied specimen, was not evident in the biopsy. In the autopsied liver specimen, necrosis comprised two areas, including acute and subacute changes.

We hypothesize that spontaneous TLS was caused by necrosis of not only tumor cells but also a large amount of nontumor cells. General awareness of spontaneous TLS is required in aggressive cases of a solid tumor when there is suspicion of organ blood supply failure.

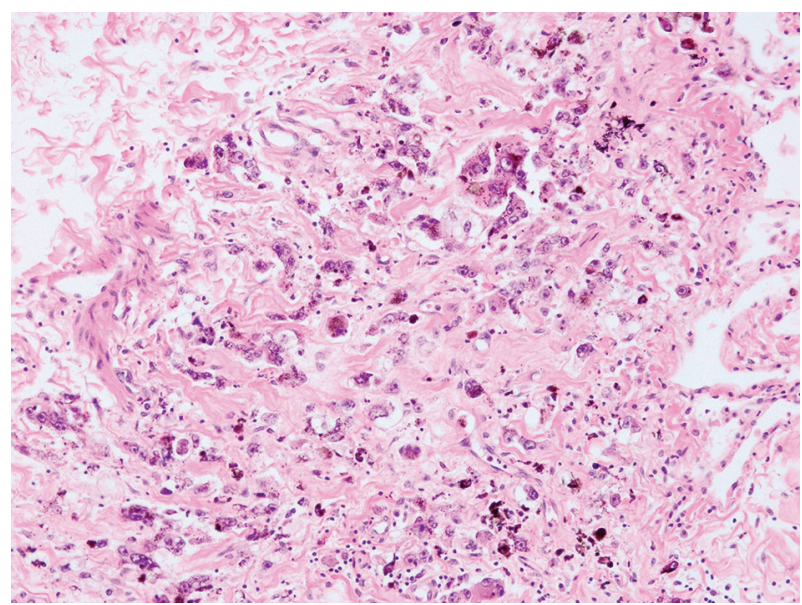

Figure 9. Microscopic view of lung lesion on biopsy. Lung metastasis without necrosis was shown.

\section{References}

1. Howard SC, Jones DP, Pui CH. The tumor lysis syndrome. N Engl J Med. 2011;364(19):1844-1854.

2. Kekre N, Djordjevic B, Touchie C. Spontaneous tumour lysis syndrome. CMAJ. 2012;184(8):913-916.

3. Coiffier B, Altman A, Pui CH, Younes A, Cairo MS. Guidelines for the management of pediatric and adult tumor lysis syndrome: an evidence-based review. J Clin Oncol. 2008;26(16):2767-2778.

4. Cairo MS, Bishop M. Tumour lysis syndrome: new therapeutic strategies and classification. Br J Haematol. 2004;127(1):3-11.

5. Hochberg J, Cairo MS. Rasburicase: future directions in tumor lysis management. Expert Opin Biol Ther. 2008;8(10):1595-1604.

6. McBride A, Westervelt P. Recognizing and managing the expanded risk of tumor lysis syndrome in hematologic and solid malignancies. J Hematol Oncol. 2012;5:75.

7. Minasian LM, Szatrowski TP, Rosenblum M, et al. Hemorrhagic tumor necrosis during a pilot trial of tumor necrosis factor -alpha and antiGD3 ganglioside monoclonal antibody in patients with metastatic melanoma. Blood. 1994;85:1055-1059.

8. Castro MP, VanAuken J, Spencer-Cisek P, Legha S, Sponzo RW. Acute tumor lysis syndrome associated with concurrent biochemotherapy of metastatic melanoma: a case report and review of the literature. Cancer. 1999;85(5):1055-1059.

9. Stoves J, Richardson D, Patel H. Tumour lysis syndrome in a patient with metastatic melanoma treated with biochemotherapy. Nephrol Dial Transplant. 2001;16(1):188189.

10. Habib GS, Saliba WR. Tumor lysis syndrome after hydrocortisone treatment in metastatic melanoma: a case report and review of the literature. Am J Med Sci. 2002;323(3):155-157.

11. Busam KJ, Wolchok J, Jungbluth AA, Chapman P. Diffuse melanosis after chemotherapy-induced tumor lysis 
syndrome in a patient with metastatic melanoma. J Cutan Pathol. 2004;31(3):274-280.

12. Borne E, Serafi R, Piette F, Mortier L. Tumour lysis syndrome induced by corticosteroid in metastatic melanoma presenting with initial hyperkalemia. J Eur Acad Dermatol Venereol. 2009;23(7):855-856.

13. Nakamura Y, Hori E, Furuta J, Ishii Y, Takahashi T, Kawachi Y, Otsuka F. Tumor lysis syndrome after transcatheter arterial infusion of cisplatin and embolization therapy for liver metastases of melanoma. Int J Dermatol. 2009;48(7):763-767.

14. Song M, Chan C, Stoeckel DA Spontaneous Tumor Ly- sis Syndrome in Matstatic Melanoma. World J Oncol. 2011;2:204-207.

15. Hande KR, Garrow GC. Acute tumor lysis syndrome in patients with high-grade non-Hodgkin's lymphoma. Am J Med. 1993;94(2):133-139.

16. Cairo MS, Coiffier B, Reiter A, Younes A. Recommendations for the evaluation of risk and prophylaxis of tumour lysis syndrome (TLS) in adults and children with malignant diseases: an expert TLS panel consensus. Br J Haematol. 2010;149(4):578-586.

17. Gemici C. Tumour lysis syndrome in solid tumours. Clin Oncol (R Coll Radiol). 2006;18(10):773-780. 